\title{
Do you know this syndrome? Nail patela syndrome: a pathognomonic dermatologic finding*
}

\section{Bruna Giusto Bunjes ${ }^{1}$}

DOI: http:/ /dx.doi.org/10.1590/abd1806-4841.20174768

\author{
Marcelle da Costa Frickmann Fernandes ${ }^{2}$
}

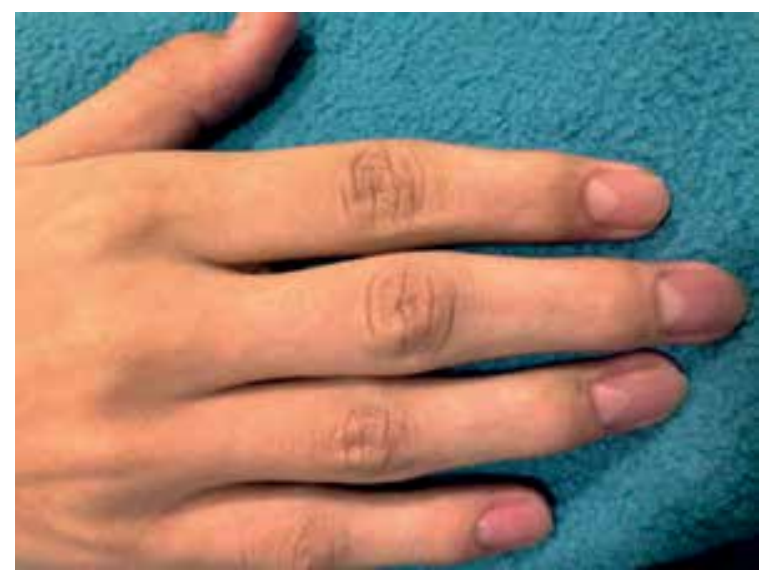

Figure 2: Triangular lunula, pathognomonic finding of Nail Patella Syndrome

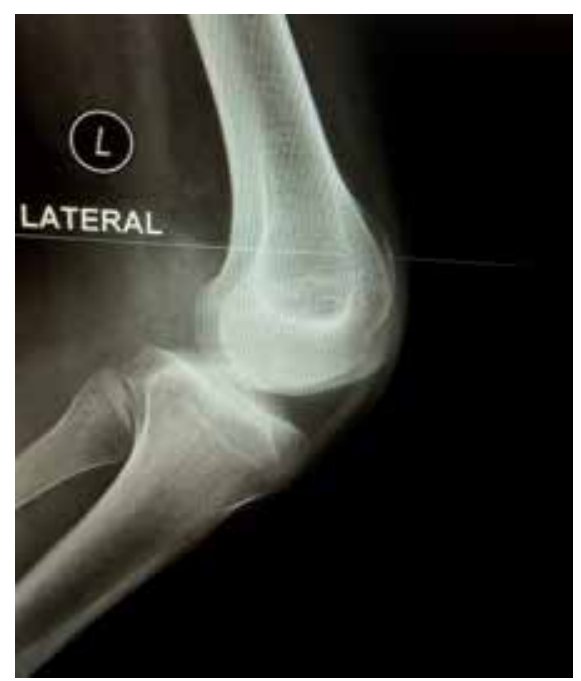

Figure 3: TX-ray of knee in profile showing patellar agenesis

Figure 1: Dystrophic nail of the 1st chirodactyl, a common finding in the Nail Patella Syndrome

Received on 28.05.2015

Approved by the Advisory Board and accepted for publication on 18.05.2015

* Study conducted in Unidade Neurointensiva do Hospital Barra D'Or - Rio de janeiro (RJ), Brazil.

Financial support: none.

Conflict of interest: none.

Department of Clinical Medicine of the Escola Paulista de Medicina - Universidade Federal de São Paulo (UNIFESP) - São Paulo (SP), Brazil.

2 Intensive Care Department of the Hospital Barra D'Or - Rio de Janeiro (RJ), Brazil.

@2017 by Anais Brasileiros de Dermatologia 


\section{DISCUSSION}

Nail patella syndrome (NPS) is a disease of autosomal dominant inheritance, occurring in 1 to 50,000 individuals. Also known as hereditary onycho-osteodysplasia, Turner-Keizer syndrome or Fong's disease, it is composed of the tetrad: patellar changes, nail changes, iliac horns and elbows anomalies. Its diagnosis is purely clinical, with the possibility of genetic testing for LMX1B mutation. It presents two pathognomonic findings: triangular lunula $(80 \%)$ and bilateral iliac horns on pelvic radiography $(70-80 \%){ }^{1}$

At physical examination, anomalies in the patella correspond to hypoplasia or aplasia in more than $90 \%$ of cases, and it is possible to identify dystrophic fingernails or triangular lunula, and the feet are rarely affected. ${ }^{1-2}$ The phenotype of the syndrome is a longilineal, emaciated individual, with muscular atrophy and difficulty in gaining weight. NPS includes vasomotor manifestations, such as cold sensation despite high temperatures. Neurologic manifestations are reported by $25 \%$ of the patients, such as decreased sensitivity to pain or temperature and intermittent paresthesia without precipitating factors. ${ }^{2}$

Possible complications of the syndrome are reported. Kidney involvement is the most serious, occurring in $30-50 \%$ of pa- tients and typically presents with hematuria and proteinuria, and in $5-10 \%$ of cases, it may develop with end-stage renal disease. ${ }^{3}$ Studies describe that the LMX1B mutation impairs the development and functioning of podocytes and glomerular filtration slits, being strongly associated with the mechanism of renal damage. ${ }^{3-4}$ Another consequence of the disease possibly associated with the LMX1B mutation is glaucoma. ${ }^{5}$

Analyzes of the LMX1B mutation reveal a role of this gene in the development of dopaminergic and mesencephalic serotoninergic neurons. This association made it possible to elaborate the hypothesis that NPS patients have an increased risk of attention-deficit/hyperactivity (ADHD) disorder, as well as major depression, symptoms that are described by these patients. ${ }^{6}$

Therefore, the early identification of NPS is fundamental to initiate the screening and management of these complications, since this is a disease without cure. In the case reported, the dermatological finding of the triangular lunula, pathognomonic of the syndrome in question, made it possible the confirmatory investigation of the disease, the clarification of the symptoms to the patient and the approach that should be performed from this diagnosis.]

Abstract: The nail-patella syndrome involves a clinical tetrad of changes in the nails, knees, elbows and the presence of iliac horns. Nail changes are the most constant feature: absent, hypoplastic, or dystrophic. A pathognomonic finding is the presence of the triangular lunula. The diagnosis of nail-patella syndrome is based on clinical findings. In this paper we will discuss a case report of this syndrome and its relation with a dermatological finding.

Keywords: Nail-patella syndrome; Nails, malformed; Nails; Repertory: nails section

\section{REFERENCES}

1. West JA, Louis TH. Radiographic findings in the nail-patella syndrome. Proc (Bayl Univ Med Cent). 2015;28:334-6.

2. Sweeney E, Fryer A, Mountford R, Green A, McIntosh I. Nail patella syndrome: a review of the phenotype aided by developmental biology. J Med Genet. 2003;40:153-62.

3. Edwards N, Rice SJ, Raman S, Hynes AM, Srivastava S, Moore I, et al. A novel LMX1B mutation in a family with end-stage renal disease of 'unknown cause'. Clin Kidney J. 2015;8:113-9.

4. Burghardt T, Kastner J, Suleiman H, Rivera-Milla E, Stepanova N, Lottaz C, et al. LMX1B is essential for the maintenance of differentiated podocytes in adult kidneys. J Am Soc Nephrol. 2013;24:1830-48.

5. Boyer 0, Woerner S, Yang F, Oakeley EJ, Linghu B, Gribouval 0, et al. LMX1B Mutations cause hereditary FSGS without extrarenal involvement. J Am Soc Nephrol. 2013;24:1216-22.

6. López-Arvizu C, Sparrow EP, Strube MJ, Slavin C, DeOleo C, James J, et al. Increased symptoms of attention deficit hyperactivity disorder and major depressive disorder symptoms in Nail-Patella Syndrome: potential association with LMX1B loss-of-function. Am J Med Genet B Neuropsychiatr Genet. 2011;156B:59-66.

\author{
MAILING ADDRESS: \\ Bruna Giusto Bunjes \\ Avenida Rene Laclete, 430 \\ 22790-303 - Rio de Janeiro - RJ \\ Brazil \\ E-mail:brunagiustob@hotmail.com
}

How to cite this article: Bunjes BG, Fernandes MCF. Do you know this syndrome? Nail Patella Syndrome: a pathognomonic dermatologic finding. An Bras Dermatol. 2017; 92(1):273-4. 